Meta

Journal des traducteurs

Translators' Journal

\title{
Pourquoi une langue emprunte-t-elle des suffixes ? L'exemple du grec et du latin
}

\section{Anna Anastassiadis-Syméonidis}

Volume 55, numéro 1, mars 2010

Le parcours du sens : d'une langue à l'autre — Mélanges offerts à André Clas

The Way of Meaning: From a Language to Another — Collection of Articles Offered to André Clas

URI : https://id.erudit.org/iderudit/039609ar

DOI : https://doi.org/10.7202/039609ar

Aller au sommaire du numéro

Éditeur(s)

Les Presses de l'Université de Montréal

ISSN

0026-0452 (imprimé)

1492-1421 (numérique)

Découvrir la revue

Citer cet article

Anastassiadis-Syméonidis, A. (2010). Pourquoi une langue emprunte-t-elle des suffixes ? L'exemple du grec et du latin. Meta, 55(1), 147-157.

https://doi.org/10.7202/039609ar
Résumé de l'article

Afin de déterminer les raisons pour lesquelles le grec a emprunté des suffixes au latin, nous examinons, en suivant le cadre théorique de Danielle Corbin, le suffixe -(i)ár(is) < du latin -arius, par exemple dans vromiaris ['malpropre'], qui construit des adjectifs dénominaux à caractère [-savant/-soutenu]. En particulier, les adjectifs en -(i)ár(is) attribuent d'une manière permanente une qualité péjorative qui, dans le cadre de l'activité humaine quotidienne, dévie de la norme sociale d'une manière perceptible directement par les sens. Ce trait, lié à leur registre, résulte du fait que le suffixe est emprunté au latin, une langue sans prestige aux yeux des Grecs. Cette représentation stéréotypique de la latinité permet au grec de marquer les différences entre, d'un côté, le [+soutenu], l'officiel, l'objectif et, de l'autre, le [-soutenu], le quotidien, le subjectif, en conservant, dans le premier cas, les éléments d'origine grecque, et en utilisant, dans le second, des éléments empruntés. 


\title{
Pourquoi une langue emprunte-t-elle des suffixes? L'exemple du grec et du latin
}

\author{
ANNA ANASTASSIADIS-SYMÉONIDIS \\ Université Aristote de Thessalonique, Thessalonique, Grèce \\ ansym@lit.auth.gr
}

\begin{abstract}
RÉSUMÉ
Afin de déterminer les raisons pour lesquelles le grec a emprunté des suffixes au latin, nous examinons, en suivant le cadre théorique de Danielle Corbin, le suffixe-(i)ár(is) $<\mathrm{du}$ latin -arius, par exemple dans vromiaris ['malpropre'], qui construit des adjectifs dénominaux à caractère [-savant/-soutenu]. En particulier, les adjectifs en -(i)ár(is) attribuent d'une manière permanente une qualité péjorative qui, dans le cadre de l'activité humaine quotidienne, dévie de la norme sociale d'une manière perceptible directement par les sens. Ce trait, lié à leur registre, résulte du fait que le suffixe est emprunté au latin, une langue sans prestige aux yeux des Grecs. Cette représentation stéréotypique de la latinité permet au grec de marquer les différences entre, d'un côté, le [+soutenu], l'officiel, l'objectif et, de l'autre, le [-soutenu], le quotidien, le subjectif, en conservant, dans le premier cas, les éléments d'origine grecque, et en utilisant, dans le second, des éléments empruntés.
\end{abstract}

\section{ABSTRACT}

In order to determine the reasons that lead Greek to borrow suffixes from Latin, we examine the suffix -(i)ár(is) < Latin -arius, e.g., vromiaris ['dirty'], within the theoretical morphological framework of Danielle Corbin. This suffix creates denominal adjectives bearing the [-learned/-elevated] feature attributing in a permanent way a degrading or below the social norm quality in a way that can be perceived by the senses, along with the use of these adjectives in everyday life situations. Their [-learned] character is a result of their having borrowed from Latin, a low prestige language, in the Greek judgment. This stereotypical representation of Latinhood allows Greek to signal the difference between the [+learned] (the official, the objective) and the [-learned] (the subjective, that used in everyday life) while keeping in the first case the elements of Greek origin, and in the second case using borrowed elements.

\section{MOTS-CLÉS/KEYWORDS}

emprunt de suffixe, adjectif dénominal, péjoration, prestige linguistique, stéréotype suffix borrowing, denominal adjective, pejoration, linguistic prestige, stereotype

\section{Introduction}

Dans le présent article, nous tenterons de déterminer les raisons pour lesquelles la langue grecque a emprunté des suffixes au latin, alors que ces suffixes sont déjà couverts par des équivalents dans la langue grecque. En particulier, en partant du cadre théorique de Danielle Corbin, nous examinerons un suffixe que le grec (L1) a emprunté au latin (L2), à savoir le suffixe -ári(os), -ár(is), $\underline{-(i) a ́ r(i s)}^{1}<$ du latin -arius, par exemple dans vivliothikarios ['bibliothécaire'], pismataris ['têtu'], klapsiaris ['pleurnichard'], pour former des adjectifs dénominaux. Ces adjectifs peuvent, par la suite, être substantivés par conversion (o peismataris ['le têtu']), le déterminant o étant le marqueur de substantivation. 
Dans un premier temps, nous examinerons ce suffixe sous un angle morphologique et sémantique, en étudiant la règle de construction des lexèmes (RCL) et le sens de ses dérivés, qui est stratifié, dans la mesure où il résulte du sens de la RCL, du sens de la base et de l'instruction sémantique du suffixe.

Dans un second temps, nous examinerons ce suffixe en comparaison de suffixes concurrents, puisqu'ils appartiennent au paradigme morphologique de la même RCL, afin d'expliquer les raisons de cet emprunt. En particulier, nous nous pencherons sur les adjectifs dérivés sous l'angle de leur registre [+/-soutenu] et de leur genre discursif (fthisikos - htikiaris ['tuberculeux'], vromikos ['sale'] - vromiaris ['malpropre'],

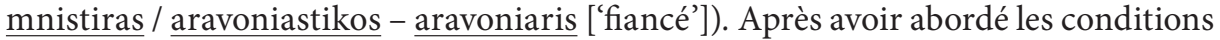
de l'emprunt d'un suffixe, nous proposerons une interprétation de l'emprunt au latin de ce suffixe, basée sur son utilisation dans la langue ${ }^{2}$.

\section{Le suffixe emprunté: -ári(os), -ár(is), -(i)ár(is) $(<-a r i u s)^{3}$}

$\mathrm{Au}$ cours des derniers siècles de l'ère préchrétienne, et plus particulièrement aux premiers siècles de notre ère, en marge des emprunts directs (porta ['porte']) ${ }^{4}$, la langue grecque a progressivement emprunté un nombre important de suffixes au latin $^{5}$, tels que -arius, -ensis, -atus, -ianus, -inus, -ullum, etc. Nous nous pencherons sur un de ces suffixes: - $\dot{\alpha} \rho \iota(o \varsigma),-\alpha \dot{\alpha} \rho(\iota \varsigma) /-\dot{\alpha} \rho(\eta \varsigma),-(\iota) \dot{\alpha} \rho(\eta \varsigma)<$-arius.

Le suffixe -arius est un exemple très représentatif, non seulement parce qu'il constitue l'occurrence la plus fréquemment attestée d'un emprunt de suffixe dans la langue grecque postclassique en raison de sa présence croissante dans les textes sur papyrus (Filos 2008: 269-273), mais aussi parce qu'il révèle une productivité élevée non seulement dans la langue grecque médiévale, sous des formes (et orthographes)

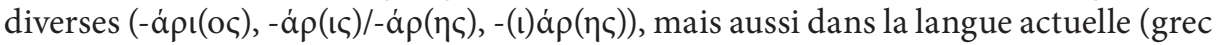
moderne).

L'analyse que nous proposons ${ }^{6}$ suit le cadre théorique défini par Corbin (1987; 1991 ; à paraître). Celui-ci est fondé sur le principe de l'associativité, en vertu duquel les RCL créent à la fois le sens et la structure des lexèmes dont le sens est compositionnel en fonction de leur structure.

Le suffixe latin -arius est utilisé pour créer des adjectifs qui se construisent sur une base verbale ou plus fréquemment nominale (postularius ['celui qui demande/exige'] ou (urceus) aquarius ['gourde']) (Miller 2006: 140-141; Filos 2008: 263). Ces adjectifs sont substantivés par conversion et se rapportent à l'auteur d'une action ou plus fréquemment à celui qui exerce une profession (lapidarius, macellarius) (Filos 2008).

Dans les papyrus grecs, le suffixe -ári(os) est présent dès le premier siècle après J.-C., non seulement comme désinence des emprunts directs au latin (arghentarios), mais également comme suffixe de mots grecs dérivés (mihanarios ['ingénieur']) (Palmer 1945: 7, note 1, 32, 48-49; Cavenaile 1952: 201-202; Filos 2008: 103). Ces dérivés sont adaptés aux règles de la phonologie et de la morphologie grecques. Au cours de cette phase, le suffixe -ári(os) est encore très proche de son ancêtre latin d'un point de vue phonologique et morphologique, mais aussi sémantique, car il sert à construire des adjectifs déverbaux ou, plus fréquemment, dénominaux (ahurarios ['celui qui travaille dans une grange']), qui peuvent être substantivés par conversion (uposhesarios ['collecteur d'impôts']), et renvoient à une personne exerçant un métier (klivanarios ['allumeur de four']). 
Le sens de la forme -ári(os) du suffixe est resté stable (Filos 2008: 269), mais la forme -(i)ár(is) ${ }^{7}$ a évolué (Anastassiadis-Syméonidis 2000a). Par ailleurs, le caractère dynamique des emprunts est étroitement lié au développement diachronique de la L1 (Gadjeva 2008: 146). En particulier, en grec moderne, on distinguerait trois catégories sémantiques :

a) des adjectifs en -ár(is) dérivés de nombres (dhekapentaris ['âgé d'environ quinze ans']), triantaris ['(personne) trentenaire']);

b) des adjectifs en -ár(is) qui, en surface, apparaissent comme des noms de profession (agheladharis ['vacher'], varkaris ['batelier']);

c) des adjectifs en -(i)ár(is) utilisés pour décrire une personne (kokaliaris ['personne osseuse']), kitriniaris ['personne au teint cireux'], psoriaris ['personne galeuse']; voir aussi Hadzidakis 1907: 611).

Selon cette classification, on pourrait avancer qu'il existe au moins deux suffixes en grec moderne, -ár(is) et -(i)ár(is) (Christofidou, Doleschal et al. 1990-1991: 73), s'ils n'étaient quelques exemples de noms de professions se terminant en -iár(is) sans que le -i puisse être considéré comme un élément de la base (karvoun-iaris ['charbonnier']), ainsi que quelques adjectifs décrivant des personnes, se terminant en -ár(is) (pismat-aris ['têtu']). Toutefois, il ne fait aucun doute qu'en grec moderne, en dépit d'un ancêtre commun (toutes les formes provenant du suffixe latin -arius), un constat s'impose: d'une part, la forme -ári(os) du suffixe se destine à la description des noms de métiers dans le registre écrit formel (vivliothikarios ['bibliothécaire']), et, d'autre part, les formes -ár(is) et -(i)ár(is) se retrouvant dans les noms et adjectifs du registre [-soutenu] (Anastassiadis-Syméonidis et Fliatouras 2004), seule la forme -(i)ár(is) est disponible pour former des adjectifs décrivant les personnes. En outre, le suffixe -ár(is) n'est plus disponible pour former des adjectifs ou des noms de profession ou de description, mais constitue la seule forme disponible pour construire des adjectifs sur des bases numérales (triantaris ['(personne) trentenaire']).

En ce qui concerne l'analyse sémantique de ces adjectifs, le suffixe -(i)ár(is) sert à construire des adjectifs établissant une relation permanente ${ }^{8}$ de possession entre la base nominale ${ }^{9}$ de l'adjectif en -(i)ár(is) et le nom recteur. La base nominale se rapporte à :

a) des maladies ou des symptômes de par leur nom commun (vlogia ['variole']), mais également l'hyperonyme arostia ['maladie'];

b) des imperfections physiques externes (fakidhes ['taches de rousseur']), ou des traits de caractère qui s'expriment par un comportement jugé socialement inacceptable (g(k)rinia ['grogne']);

c) un élément ou un trait qui déprécie l'apparence physique au vu des normes sociales (koureli ['loque']).

Le trait qu'attribue l'adjectif en -(i)ár(is) au nom recteur est clairement perçu par les sens, particulièrement la vue (spiriaris ['boutonneux']), mais aussi l'ouïe (vlastimiaris ['personne qui invective']), l'odorat ou une combinaison de sens (vromiaris ['individu malpropre']). Lorsque ce trait s'applique au comportement humain, il est également perçu par les sens à travers les actes du référent du nom recteur, par ex., zimiaris ['individu qui cause des dégâts'], psihoponiaris ['personne compatissante']. Nous considérons que le trait quelque peu péjoratif ou inférieur à la norme sociale attaché à l'utilisation de ce suffixe constitue le lien entre les adjectifs de profession et ceux décrivant des qualités construits au moyen du suffixe -(i)ár(is): les métiers 
concernés correspondent tous à un statut social inférieur (varkaris ['batelier'], arkoudhiaris ['montreur d'ours'], maimoudhiaris ['montreur de singe'], skoupidhia-

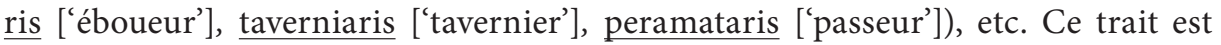
inclus dans l'instruction sémantique du suffixe -(i)ár(is), qui renvoie toujours à l'élément péjoratif d'une qualité par rapport à une norme. En conséquence, le suffixe -(i)ár(is) sert à la construction d'adjectifs qui attribuent d'une manière permanente un caractère péjoratif identifiable par les sens. Le locuteur qui utilise un adjectif dérivé en -(i)ár(is) émet simultanément un jugement péjoratif sur le référent du nom recteur $^{10}$. C'est dans l'instruction sémantique du suffixe -(i)ár(is) que se trouve le trait distinctif [+ péjoratif] qui permet d'utiliser ces dérivés dans certaines situations comme injures, souvent au vocatif, par ex. klapsiari! ['pleurnichard!'].

Ceci signifie que les adjectifs et les noms construits au moyen du suffixe -(i)ár(is) sont par nature axiologiques, car ils sont marqués, d'une manière permanente, par le trait [+péjoratif] inclus dans leur sens. Le trait distinctif [+péjoratif] est un hyponyme de l'hyperonyme [subjectif] (Kerbrat-Orecchioni 1980). Les dérivés en -(i)ár(is) sont doublement subjectifs, dans la mesure où ils reflètent, d'une part, l'idéologie du locuteur et, d'autre part, le fait que le locuteur prend position contre le référent (voir aussi Kerbrat-Orecchioni 1980: 91). En conséquence, le suffixe-(i)ár(is), de par son caractère subjectif, n'a pas sa place dans un discours objectif, par exemple scientifique, dans lequel le locuteur s'efforce précisément de faire preuve d'objectivité. Par contre, les dérivés en -(i)ár(is) apparaissent dans les genres à caractère subjectif, par exemple un discours polémique, dans lequel le locuteur exprime son opinion et son idéologie de manière assez directement ou indirectement ouverte, afin de rabaisser ou d'attenter au prestige de son adversaire. C'est pourquoi ces adjectifs ou ces noms sont destinés aux situations de discours informel, dans le registre familier et dans la langue orale.

\section{Pourquoi emprunter un suffixe?}

Différents paramètres comme le temps, les canaux linguistiques par lesquels un emprunt a été importé, le registre, ainsi que d'autres facteurs, jouent un rôle important dans le phénomène de l'emprunt. Il existe souvent des différences de registre entre les discours familier et soutenu ainsi qu'entre le langage parlé et écrit.

Examinons deux raisons extralinguistiques et une raison linguistique qui ont facilité l'introduction de suffixes latins dans la langue grecque:

a) Le temps: période hellénistique;

b) Les conditions: la cohabitation dans l'Empire romain, interaction historique. Le latin était la langue officielle de l'empire, mais le grec a conservé un statut semiofficiel dans sa partie orientale, en raison de la politique linguistique des Romains généralement favorable au grec, tout au moins dans cette partie de l'empire (Coleman 2007: 799; Adams 2003: 540-541, 555, 754);

c) La distance typologique: le grec et le latin ont des structures typologiques congruentes (similitudes dans les systèmes de flexion et de dérivation) en raison de leur origine commune indo-européenne. À cet endroit, il existe toutefois un effet disproportionné: l'influence du grec sur le latin a été plus pénétrante et a eu un impact plus durable. Ce phénomène est attribuable à plusieurs raisons: le nombre réduit de locuteurs latins en Orient, l'attitude des Grecs face aux langues barbares, la politique linguistique des Romains, etc. 
La littérature attribue le nombre relativement faible d'emprunts grecs au latin à l'attitude de dénigrement des Grecs à l'égard du latin et de ses locuteurs. Il existe toutefois un autre facteur: les emprunts de la langue grecque au latin sont de deux types: d'une part, les emprunts originaires de la terminologie administrative dans les documents officiels (administration, tribunaux, armée, système juridique) et les ouvrages littéraires de registre soutenu (séculaires et religieux) liés à l'Empire romain officiel, et, d'autre part, les emprunts au vocabulaire quotidien. Il existe un rapport direct entre le vocabulaire quotidien et le caractère [-soutenu] de ces adjectifs. Les changements intervenus dans la vie politique lors de la fondation de l'Empire romain d'Orient ont abouti à l'abandon des emprunts du premier type; parallèlement à l'attitude de dénigrement des Grecs à l'égard du latin, les emprunts du deuxième type sont devenus des mots au caractère moins prestigieux, principalement utilisés dans la langue grecque familière (Anastassiadis-Syméonidis 1994: 104-105).

Seuls quelques mots de la langue quotidienne ont réussi à traverser l'époque médiévale et sont parvenus jusqu'à nous. Ce fait tend à indiquer un degré différent d'intégration du vocabulaire du registre familier par rapport aux termes du registre soutenu: les termes administratifs, politiques et militaires sont plus étroitement liés aux superstructures sociales; une fois intervenus les changements historiques (politiques, socio-économiques, culturels) qui ont affecté ces structures, principalement la transformation progressive et le déclin de l'Empire romain d'Orient, une grande partie du vocabulaire concerné est devenu obsolète. La disparition de nombreuses formes soutenues latines s'explique, en outre, par les efforts conscients puristes entrepris par l'état byzantin à partir du $\mathrm{x}^{\mathrm{e}}$ siècle après J.-C. pour helléniser la terminologie et la nomenclature administratives (Kahane et Kahane 1982: 133). Les dérivés grecs renvoient généralement aux professions des classes sociales inférieures (ahurarios, tapitarios), par opposition aux emprunts qui concernent les fonctions militaires et administratives (kagkelarios, leghionarios, notarios). Ultérieurement, au cours des époques byzantine et moderne, le suffixe se retrouve dans des noms de professions inférieures (upodimatarios ['vendeur de chaussures']) et intermédiaires/supérieures (spatharios ['membre de la garde impériale de l'Empire byzantin']), mais son utilisation s'étend désormais aux adjectifs de qualité tels que psoriari(o)s ['galeux'] (Jannaccone 1950: 57). Les seules formes de racines grecques relatives aux classes supérieures concernent les professions inférieures/intermédiaires administratives et juridiques telles que nomikarios ['avocat, notaire']. À partir de l'époque byzantine, le suffixe apparaît sous la forme -ár(is), ex. portaris < portarius (Dieterich 1928: 111-112; Filos 2008: 268; Jannaccone 1950: 56). Les emprunts latins en -ári(os) <-arius ont trait à la classe supérieure. Les mots à racine grecque en -ári(os) ont trait à la classe inférieure. Les suffixes possédant une signification technique sont probablement passés dans la langue grecque par les institutions romaines (armée, administration) qui ont influencé les locuteurs grecs ayant des contacts fréquents (apparemment soldats et personnel administratif) (Adams 2003: 495). Ce suffixe révèle une préférence pour une catégorie particulière de mots grecs (essentiellement les mots de registre familier) (Filos 2008: 301).

Selon notre analyse, fondée sur le sens des adjectifs en -(i)ár(is), les emprunts du grec au latin symbolisent une civilisation considérée comme inférieure à la civilisation grecque. Même s'ils ne se différencient pas du grec du point de vue phonologique et morphologique, ces emprunts possèdent des particularités sémantiques 
et pragmatiques qui révèlent leur origine inférieure, selon les critères des Grecs. Il en va de même pour les autres suffixes ${ }^{11}$ grecs empruntés au latin -át(os) $<$-atus, -isi(os) $<$-ensis, -oúr(a) $<$-ura, -oúkl(a) $<$-ucla $<$-cula, -poul(os) $<$-pullus, dont nous identifions le caractère [-soutenu ${ }^{12}$. En particulier, nous considérons que leur caractère [-soutenu] résulte du fait qu'ils sont empruntés au latin et qu'ils sont utilisés dans les situations de la vie quotidienne. Étant donné que chaque élément du langage est le reflet de la civilisation de ses locuteurs, chaque emprunt de la L2 crée dans la L1 une représentation de cette civilisation. Le stéréotype qui résulte de cette représentation devient partie intégrante du champ sémantique de ces emprunts dans la L1 et reflète de manière sous-jacente les opinions des locuteurs de la L1 à la fois pour la civilisation des locuteurs de la L2 et pour leur propre civilisation (voir aussi Sarale 2008: 165). On constate la prégnance du stéréotype interculturel: le stéréotype dénué de prestige, péjoratif, se référant à la vie quotidienne, qui contribue à la définition de l'ethnotype latinité (voir aussi Sarale 2008: 155).

De plus, si l'on opère une distinction entre les catégories des experts, des savants, et les catégories populaires (Taylor 1989: 72), il devient clair que les noms de base de toutes les catégories, même s'ils possèdent des définitions de type expert - c'est-à-dire élaborées par des scientifiques - provenant d'une catégorisation basée sur des conditions nécessaires et suffisantes, contribuent à la construction de ces dérivés sur la base de leur définition populaire, structurée à partir d'éléments prototypiques; leur création découle de la manière dont les individus perçoivent les objets de leur environnement et de l'effet exercé sur eux par ces objets. Nous pensons que le sens prédictible des adjectifs dérivés au moyen du suffixe -(i)ár(is) résulte des traits sémantiques de leur nom de base et de l'instruction sémantique du suffixe: un sens de profession ou de qualité est évoqué, à travers un prisme de rapports culturels. Pour construire un adjectif à partir de ce suffixe, il doit y avoir compatibilité morphologique, sémantique et pragmatique avec le nom de base et avec le nom recteur.

En effet, le suffixe -(i)ár(is) permet la construction d'adjectifs qui renvoient d'une manière permanente à une connotation péjorative liée au métier, à l'anatomie ou au comportement, considérés en dessous de la norme établie, alors que le locuteur exprime simultanément un jugement péjoratif sur le référent du nom recteur (vromiaris anthropos ['personne malpropre']). De ce fait, si le grec moderne possède deux formes pour un nom, une forme [+soutenue] et une forme [-soutenue], le suffixe -(i)ár(is) s'applique aux formes [-soutenue] ou [+/-soutenue] ${ }^{13}$ (htikiaris ['tuberculeux'], ${ }^{*}$ fthisiaris $/{ }^{*}$ fimatiaris - fthisikos, fimatikos ['tuberculeux']).

Il est particulièrement intéressant de constater qu'il existe en grec moderne des paires de mots ${ }^{14}$ comme htikiaris ['tuberculeux'] - fthisikos/fimatikos ['tuberculeux'], arostiaris ['souffreteux'] - filasthenos ['maladif'], qui diffèrent non seulement par l'instruction sémantique du suffixe, mais aussi par le trait [+/-soutenu] et, partant, le genre de discours dans lequel ils apparaissent. Il existe des différences pragmatiques entre les deux membres des paires de mots ci-dessus (Anastassiadis-Syméonidis 1994: 104-105). En effet, les adjectifs construits au moyen du suffixe -(i)ár(is) n’apparaissent ni dans les textes scientifiques, ni dans la terminologie afférente. En conséquence, différents suffixes appliqués à une même base peuvent en sélectionner différents traits; c'est pour cette raison que les adjectifs des paires ci-dessus ne peuvent être considérés comme synonymes ${ }^{15}$. 
De même, ce modèle nous permet non seulement d'expliquer les similitudes et les différences sémantiques et pragmatiques entre les mots considérés à tort comme synonymes, mais aussi de prédire certaines restrictions. Nous pouvons en particulier expliquer:

a) pourquoi il est possible d'avoir des adjectifs dérivés d'un même nom de base, mais porteurs d'un suffixe différent (vromikos ['sale'] - vromiaris ['malpropre']): -(i)ár(is) attribue une qualité permanente dans un registre [-soutenu], alors que -ik(os) construit des adjectifs signifiant ['qui a un rapport avec le nom de base'] dans un registre [+/-soutenu] et [+soutenu]. De ce fait, nous sommes en mesure d'expliquer pourquoi les adjectifs à désinence en -ik(ós) et en -(i)ár(is) ne sont pas synonymes;

b) pourquoi certains suffixes ne s'appliquent pas à certains noms de base, en raison d'une exigence de compatibilité. Les adjectifs dérivés en -ik(ós) préfèrent l'allomorphe [+soutenu] du nom de base, alors que les adjectifs en -(i)ár(is) sont construits à partir de l'allomorphe [-soutenu] du nom de base (fthisikos ['tuberculeux'] - htikiaris (

\section{Conclusion}

Le point essentiel de la recherche sur l'emprunt d'un suffixe est, d'une part, l'analyse diachronique visant à retrouver la forme d'origine dans la L2 et, de l'autre, l'analyse synchronique pour identifier le rôle sémantique que ce suffixe joue dans la L1; pour nous, il est tout aussi essentiel de retrouver les raisons qui ont conduit la L1 à emprunter ce suffixe.

Le choix du cadre théorique de Danielle Corbin nous a permis d'unifier l'instruction sémantique du suffixe que nous avons analysé et de découvrir son rôle sémantique: il sert à construire des adjectifs dénominaux à caractère [-soutenu]. En particulier, les adjectifs en -(i)ár(is) attribuent, d'une manière permanente, une qualité péjorative, qui dévie de la norme d'une manière perceptible directement par les sens, dans le cadre de l'activité humaine quotidienne. Ce modèle nous a permis non seulement d'expliquer les similitudes et les différences sémantiques et pragmatiques entre les mots considérés à tort comme synonymes, mais, en outre, de prédire certaines restrictions. De même, ce modèle nous a permis de comprendre mieux les mécanismes qui déclenchent l'emprunt de suffixe.

Emprunter constitue pour une langue un moyen sûr d'introduire des nuances plus fines aux niveaux sémantique et pragmatique. C'est précisément la méthode choisie par la langue grecque: en empruntant au latin le suffixe -(i)ár(is), le grec a acquis l'outil grammatical permettant de marquer les différences entre, d'une part, le [+soutenu], l'officiel, l'objectif et, de l'autre, le [-soutenu], le quotidien, le subjectif, en conservant, dans le premier cas, les éléments d'origine grecque, et en utilisant, dans le second, des éléments empruntés (mnistiras/aravoniastikos - $\underline{\text { aravoniaris }}$ 'fiancé'). En utilisant ses propres outils, la langue grecque démontre la valeur de la parole d'Horace: "Grocia victa ferum victorem vicit et artis et litteras agresti Latio intulit $^{16}$.» 


\section{NOTES}

1. Les désinences à l'intérieur des parenthèses à la droite d'un mot indiquant le genre grammatical, le cas et le nombre, n'interviennent pas dans le processus de dérivation morphologique.

2. Un aspect de ce problème est lié à la relation entre les formes -ári(os), -ár(is), -(i)ár(is). Dans la Grammaire du grec moderne (démotique) (Triantaphyllidis $1941 \$ \$ 286,298)$ et la Grammaire $d u$ grec moderne (Tsopanakis 1994: 668), ce suffixe apparaît avec deux allomorphes distincts, -ár(is) et -(i)ár(is). Cette unification semble être basée sur l'étymologie, car le latin -ari(u)s a donné -ári(os) en grec (skoutarios $<$ scutarius, dont vient -ár(is) (ghounaris $<$ gunnarius, fournaris $<$ furnarius, kavalaris $<$ kavalarios $<$ caballarius), et -(i)ár(is) (silentiarios $<$ silentiarius). Dans ce dernier cas, le -i de gauche a été intégré au suffixe, bien qu'il s'agisse d'une voyelle de la base nominale (voir aussi Hatzidakis 1905: 421 ; 1907: 527, 582; Triantaphyllidis 1909: 431, 433; Filintas 1925: 119-121, 130; Petrounias 1991 pour une analyse approfondie du suffixe -aría). Cette voyelle latine correspondait soit au i (silentiarios < silentiarius) soit au e (langkiarios $<$ lancearius). La question se pose donc de savoir si, d'un point de vue synchronique, nous sommes en présence de variantes d'un même suffixe ou de deux suffixes, voire plus. La réponse proposée se fonde sur des critères de sens et de disponibilité.

3. Sur environ 2900 emprunts au latin qui, selon Viscidi (1944: 58), sont passés dans la langue grecque (d'après analyse des sources littéraires), on considère qu'environ $10 \%$ d'entre eux survivent en grec moderne (Filos 2009).

4. Pour une vue d'ensemble de la bibliographie sur les emprunts au latin dans la langue grecque, voir Filos (2008). Concernant les emprunts de suffixes en particulier, consulter Palmer (1945) et Cavenaile (1952), qui abordent les thèmes généraux de la morphologie des emprunts au latin dans les textes grecs sur papyrus.

5. Selon Palmer (1945: 7), il existe plus de trente mots grecs formés à partir du suffixe latin -ári(os), et plus de soixante-dix emprunts directs.

6. Pour de plus amples informations concernant le grec moderne, voir Anastassiadis-Syméonidis $(1992 ; 1996 ; 1998 ; 2000$; 2000b; 2008; 2009).

7. Par ailleurs, le suffixe _(i)ár(is) est intéressant, car il pose le problème de la définition des limites des morphèmes, liées à l'allomorphie, mais également aux origines du morphème.

8. Exemples: les adjectifs arostos ['malade'] et arostiaris ['maladif']. Le premier décrit un état passager, alors que le second décrit une personne souffreteuse, maladive. La situation est identique pour les paires vromikos ['sale'] - vromiaris ['malpropre'] et alanis ['vagabond'] - alaniaris ['vagabond'].

9. Dans la littérature, il n'existe aucun consensus sur la catégorie grammaticale de la base à laquelle s’applique le suffixe -(i)ár(is). Selon les descriptions disponibles (par exemple, dans la Grammaire du grec moderne (démotique) [Triantaphyllidis 1941: \$298]), cette base appartient soit à la catégorie des noms (gkriniaris ['grognard'], kiliaris ['ventru'], kokaliaris ['personne osseuse'], pexnidhiaris ['joueur']), soit à celle des adjectifs (arrostiaris ['maladif']), soit à celle des verbes (klaniaris ['péteur/trouillard'], klapsiaris ['pleurnichard'], lismoniaris ['étourdi'], fovitsiaris ['peureux']). Selon Hristodoulos (2009), la base peut être nominale ou verbale. Dans une tentative d'unification de la catégorie de la base, nous pouvons considérer que la base des adjectifs en - $(l) \dot{\alpha} \rho(\eta \varsigma)$ relève de la catégorie nominale, car la base de la plupart des adjectifs de ce type y appartient. Quant aux dérivés du troisième groupe, on peut considérer qu'ils sont construits sur une base qui est un nom déverbal.

10. En raison du caractère péjoratif des mots construits au moyen du suffixe -(i)ár(is), de nombreux surnoms et, partant, des noms de famille, sont créés sur ce mode.

11. Filos $(2008: 95,254)$ ne fournit pas une explication complète de ce phénomène: «Les suffixes latins ont été introduits dans la langue grecque dans le cadre de l'adoption des emprunts latins, mais ils ont acquis ultérieurement un statut indépendant et ont été intégrés à la morphologie grecque avant d'être également suffixés aux racines grecques». Et un peu plus loin, il ajoute: «Les emprunts ont permis de former quelques suffixes, qui ont ensuite été exploités pour former de nouveaux mots à partir de racines grecques et latines » (Filos 2008: 321). Il est bien connu qu'il est difficile d'emprunter tant des éléments grammaticaux, tels que les suffixes (opposés aux éléments lexicaux), que des éléments infralexicaux (opposés aux mots) (Anastassiadis-Syméonidis 1994: 1996). Cet emprunt a été possible grâce aux trois conditions que nous avons décrites (AnastassiadisSyméonidis 1996: 102; 2009):

a) Condition morphologique: emprunts massifs du grec au latin d'adjectifs en -(i)ar(is);

b) Condition morphosémantique: emprunt simultané de la base et de l'adjectif dérivé; 
c) Condition sémantique: reconnaissance de la contribution sémantique du suffixe en raison de la deuxième condition. Â partir de ce point, le grec peut emprunter le suffixe et construire des mots dérivés qui conservent le suffixe, mais dont la base est traduite du latin selon une construction correspondante (voir aussi Tzitzilis 2001: 43).

12. Pour -isi(os), voir Anastassiadis-Syméonidis (2009).

13. Nous considérons qu'il ne s'agit pas d'une distinction binaire, mais d'une distinction s'étalant sur un continuum sur lequel on peut repérer au moins trois points remarquables: [+soutenu], [+/soutenu], [-soutenu] (Anastassiadis-Syméonidis et Fliatrouras 2004).

14. Nous ne comparerons pas -(i)ár(is) aux suffixes verbaux qui se rapportent à un métier, tels que -tir, -tor, -tis, qui appartiennent au paradigme morphologique d'une autre RCL (voir aussi Filos 2008: 268).

15. En conséquence, nous ne partageons pas l'avis de Filos (2008: 254), lorsqu'il déclare que, très souvent, des suffixes grecs, qui remplissaient les mêmes fonctions morphologiques et sémantiques, préexistaient et coïncidaient parfois phonologiquement et/ou fonctionnellement avec les suffixes latins: dans certains cas, les suffixes importés coexistaient avec les suffixes locaux et pouvaient être indistinctement appliqués aux racines grecques et latines (par exemple, mihan-ários). L'introduction, au cours de la période romaine (Filos 2008: 262), de nombreux suffixes latins masculins, comme -arius > -ári(os), dans la formation de mots du grec postclassique est un phénomène notable, particulièrement si l'on prend en considération le nombre de suffixes grecs indigènes qui remplissaient globalement des fonctions identiques ou comparables (Filos 2008: 259).

16. La Grèce vaincue vainquit le vainqueur rustre et introduisit les arts et les lettres au Latium agreste (traduction de l'auteure).

\section{RÉFÉRENCES}

AdAms, James Noel (2003): Bilingualism and the latin language. Cambridge: CUP.

AnAstassiadis-Syméonidis, Anna (1992): La dérivation morphologique selon le modèle de D. Corbin. Studies in Greek Linguistics. 13:505-526.

AnAstassiadis-Syméonidis, Anna (1994): Emprunt néologique du grec moderne. Emprunts directs du grec au français et à l'anglo-américain. Analyse morphophonologique. Thessaloniki: Université Aristote de Thessaloniki.

Anastassiadis-Syméonidis, Anna (1996): À propos de l'emprunt suffixal en grec moderne. Cahiers de lexicologie. 68(1):79-106.

Anastassiadis-Syméonidis, Anna (1998): On Modern Greek Denominal Adjectives. Proceedings of the first Mediterranean Conference of Morphology (Mytilène, Grèce, 19-21 septembre 1997). Mytilini: University of Patras, 29-40.

AnAstassiadis-Syméonidis, Anna (2000a) : Suffix -(i)ar(is) in Modern Greek. Studies in Greek Linguistics. 20:65-74.

Anastassiadis-Syméonidis, Anna (2000b): La classe des défauts humains en grec moderne. In: Lexique, Syntaxe et Sémantique. Mélanges offerts à Gaston Gross à l'occasion de son soixantième anniversaire. BuLAG. Hors-série:83-94.

AnAstassiadis-Syméonidis, Anna (2002) : Dictionnaire inverse du grec moderne. Thessaloniki: Université Aristote de Thessaloniki.

AnAstassiadis-SymÉONIDIS, Anna (2008) : Les adjectifs temporels suffixés en -in(os) et -iatik(os) en grec moderne. In: Fradin BERNARD, dir. La raison morphologique. Hommage à la mémoire de Danielle Corbin. Amsterdam: John Benjamins, 17-27.

Anastassiadis-Syméonidis, Anna (2009): Le suffixe -isi(os) en grec moderne. Studies in Greek Linguistics. 29:58-73.

Anastassiadis-Syméonidis, Anna et Fliatouras, Asimakis (2004): Le trait [+/- soutenu] en grec moderne - Définition et typologie. Proceedings of the $6^{\text {th }}$ International Conference of Greek Linguistics. Rethymno: Université de Crète, 110-120.

Anastassiadis-Syméonidis, Anna et Chatzopoulou, Aikaterini (2009): Changement de frontières morphémiques et création suffixale. In: Constantin BoBAs, dir. D'une frontière à l'autre. Mouvements de fuites, mouvements discontinus dans le monde néo-hellénique. Athènes / Lille: Éditions Gavrielidès / Septentrion, 589-600. 
Cavenaile, Robert (1952): Quelques aspects de l'apport linguistique du grec au latin. Aegyptus. 32:193-203.

Christofidou, Anastasia, Doleschal, Ursula et Dressler, Wolfgang U. (1990-1991): Gender agreement via derivational morphology in Greek. Glossologia. 9-10:69-79.

Coleman, Robert (2007): Greek and Latin. In: Anastassios-Fivos Christidis, dir. A History of Ancient Greek. From the Beginnings to Late Antiquity. Cambridge: Cambridge University Press, 792-799.

Corbin, Danielle (1987/1991): Morphologie dérivationnelle et structuration du lexique. 2 vol. Tübingen / Villeneuve d'Ascq: Max Niemeyer Verlag / Presses Universitaires de Lille.

Corbin, Danielle (1991): Introduction - La formation des mots: structures et interprétations. Lexique. 10:7-30.

Corbin, Danielle (à paraître): Le lexique construit. Méthodologie d'analyse.

Dieterich, Karl (1928): Die Suffixbildung im Neugriechischen. Balkan Archiv. 4:104-167.

Filintas, Menos (1925): Glossognosia kai glossographia elliniki. Vol 2. Athènes: Kazomari.

FILos, Panagiotis (2008): Studies in the Morphology of Latin Loanwords into Greek: Evidence from the Papyri. Thèse de doctorat non publiée. Oxford: University of Oxford.

Filos, Panagiotis (2009): Latin loanwords and their influence upon the post-classical Greek derivational morphology: the case of the suffix -apıs [in Greek]. Proceedings of the $8^{\text {th }}$ International Conference on Greek Linguistics. (Ioannina, 30 août-2 septembre 2007). Consulté le 2 janvier 2010, <http://www.linguist-uoi.gr/cd_web/docs/greek/046_filosICGL8. pdf $>$.

Gadjeva, Snejana (2008): Dynamisme de l'emprunt: les turcismes dans la langue bulgare. Neologica. 2:131-147.

Hatzidakis, Georgios (1905): Grec médiéval et moderne. Vol 1. Athènes: Sakellarios.

Hatzidakis, Georgios (1907): Grec médiéval et moderne. Vol 2. Athènes: Sakellarios.

Horrocks, Geoffrey (1997): Greek. A History of the Language and its Speakers. London: Longman.

Hristodoulou, Hristodoulos (2009): Le mot klousara de l'idiome de Kozani et sa formation. Travail de $2^{\text {e }}$ cycle dans le cadre du cours «Morphologie». Thessalonique: Université Aristote de Thessalonique.

JannacCone, Sylvie (1950): Recherches sur les éléments grecs du vocabulaire latin de l'empire. Paris: Honoré Champion.

Kahane, Henrich et Kahane, Renée (1982): The Western Impact on Byzantium: The Linguistic Evidence. Dumbarton Oaks Papers. 36:127-153.

Kerbrat-Orecchioni, Catherine (1980/1997): L'énonciation. De la subjectivité dans le langage. Paris: Masson / Armand Colin.

MéLis-Puchulu, Agnès (1991): Les adjectifs dénominaux: des adjectifs de «relation». Lexique. 10:33-60.

Meyer, Gustav (1895) : Neugriechische Studien III. Die lateinischen Lehnworte im Neugriechischen. Sitzungsberichte der kaiserlichen Akademie der Wissenschaften in Wien, Philosophischhistorische Klasse, Band 132. Wien: Wiener Akademie.

Miller, Gary D. (2006): Latin Suffixal Derivatives in English and their Indo-European Ancestry. Oxford: Oxford University Press.

Palmer, Leonard Robert (1945) : A Grammar of Post-Ptolemaic Papyri. I1 : The Suffixes. London: Oxford University Press.

Petrounias, Evangelos (1991): Le suffixe néohellénique -aria emprunté au vénitien. Sources d'emprunt multiples et réemprunt. Studies in Greek Linguistics. 11:53-69.

Psaltes, Stamatios B. (1913): Grammatik der byzantinischen Chroniken. Göttingen: Vandenhoeck \& Ruprecht.

SARAle, Jean-Marc (2008): Processus de lexicalisation de quelques emprunts au japonais. Neologica. 2:149-167.

TAYLOR, John R. (1989) : Linguistic Categorization-Prototypes in Linguistic Theory. New York: Oxford University Press. 
Triantaphyllidis, Manolis (1909/1963): Die Lehnwörter der Mittelgriechischen Vulgärliteratur. Euvres complètes de Manolis Triantaphyllidis. Vol. 1. Thessaloniki: Institut d'Études Néohelléniques, 299-494.

Triantaphyllidis, Manolis (1941/1978): Grammaire du grec moderne (démotique). Athènes / Thessaloniki: OESB / Institut d'Études Néohelléniques.

Tsopanakis, Agapitos (1994): Grammaire du grec moderne. Thessaloniki: Kyriakidis et Estia. Tzitzilis, Christos (2001): Methodische Bemerkungen zu den Lehnübertragungen in den Balkansprachen. Balkansko Ezikoznanie-Linguistique Balkanique. 41(1):41-53.

VIscidi, Federico (1944): I prestiti latini nel greco antico e bizantino. Florence: Casa Editrice Dott. Antonio Milani. 